\title{
The basics of formation of expert systems for industry and science
}

\author{
Igor G. Vidyaev ${ }^{1}$, a , Maksim I. Pushkarev ${ }^{1}$, Nikita V. Martyushev ${ }^{1}$, Mikhail A. Sirenko ${ }^{2}$, Ju. A. Kozlikina ${ }^{1}$ \\ ${ }^{1}$ Tomsk polytechnic university, 634050, Lenina Avenue, 30, Tomsk, Russia \\ ${ }^{2}$ Tomsk State University, 634050, Lenina Avenue, 36, Tomsk, Russia
}

\begin{abstract}
This paper considers the basics of development of a practice-oriented information system of examination of industrial and scientific projects realized in the field of machine building. The first part of the paper is devoted to the main problems which are necessary to solve while designing such systems. The methods of self-organisation and adaptation, the use of expert evaluations and other approaches, for instance, those connected with the study of various fundamental and applied aspects of intelligence control systems, were considered. The main problems that are solved when creating an effective system of the expert selection were structured and expounded. In addition, the information, based both on already existing models (DFD, BPMN) and on modern approaches (a system approach, Delphi technique, artificial neural networks), concerning the creation of an effective system of expert evaluations was presented. A combination of the approved methods and new approaches allows adapting the system to different conditions of use.
\end{abstract}

\section{Introduction}

Most of the modern studies on the problems of development of information systems of expert evaluation base on the learned works of Russian and foreign scientists, connected with two basic trends. The first one is the methods of objects control on the basis of principles of self-organization and adaptation. Such scientists as R. Bellman, S. Beer, K. Glover, A.N. Guda, A. G. Ivakhnenko, M.B. Ignatyev, R. Kalman, A. A. Kolesnikov, N.N. Lyabakh, G. Saridis, H. Haken, V.V. Tsyganov, W.R. Ashby have made a major contribution to the study of this trend. And the second trend represents a solution of the problems of application of expert evaluations in control problems. Here the outstanding contribution was made by such scientists as G.G. Azgaldov, S.D. Beshelev, F.G. Gurvich, L.G. Yevlanov, V.A. Kutuzov, E.P. Rayhman, A.A. Frenkel, D.S. Shmerling. Besides these two trends scientists use other approaches that are connected, for example, with the study of different fundamental and applied aspects of intelligence control systems (N. Viner, V.M. Glushkov, D.I. Dubrovsky, S.M. Kovalev, A.A. Lyapunov, M. Minsky, D.A. Pospelov, F. Rosenblatt, V.B. Tarasov, A.N. Shabelnikov).

The information systems of expert evaluation, created on the basis of the works of the aboveenumerated scientists, have been applied in very diverse problem domains such as finances, oil and gas industry, power engineering, transportation, pharmaceutical production, space exploration, chemistry, education, telecommunication and connection and others, for instance, MIXER (the expert system, used in the field of computer systems, which assists programmers in microprogrammes coding for very-large-scale integration circuit (VLIC) TI990 developed by Texas Instruments), $\mathrm{ACE}$ (the expert system, used in the electronics, which detects failures in a telephone network and gives recommendations on the needed repair and rehabilitation measures), I\&W (the expert system, used in the military sphere, which assists the analysts, working in an intelligence service, in predicting when and where the next armed conflict will take place), FALCON (the expert system, used in the sphere of processes control at an enterprise, which identifies probable causes of process parameters aberration at a chemical plant, interpreting data consisting of numerical values of instruments readings, switches positions and conditions of emergency sensors), Family doctor (the simple medical expert system which determines the nature of an illness, basing on the responses of a user, obtained as a result of the dialogue) and in many other fields. The sphere of scientific expertise and industrial project expertise has not become an exception to the rule. In this case there is as well a multitude of research works on creation of methods and expert systems. However, the rapid development of science and industry, emergence of new technologies and trends, improvement

\footnotetext{
$\overline{{ }^{a} \text { Corresponding author: vig@tpu.ru }}$
} 
of the overall well-being of society demand new approaches and new methods allowing making a selection of projects in conformity with time.

\section{Materials and methods}

Modern tendencies of development of information provision of scientific research and design projects require availability of expert systems of operative evaluation of their prospectivity and expediency of conducting and realization [1], allowing quick analysis and processing of a significant amount of information obtained from different fields of science and technology. For example, a lot of scientific works in the sphere of mechanical engineering are interdisciplinary and they simultaneously involve designers, technologists, physicists and chemists, programmers and economists. Specialists from different scientific branches use their own specific terms, which are not always comprehensible for colleagues. The capabilities of the information systems of expert evaluation, being implemented nowadays, such as ASEE (automated system of expert evaluation) "Selection of a project", EXCO, COMBI-PC, ASEE-8 and others, are sufficiently restricted. They should be adjusted to the problems of evaluation of scientific projects taking into consideration the peculiarity of the field of research, which, as a rule, is prohibited by the developers of the system; the capabilities of supplementing, updating, error corrections are extremely narrow. This fact creates significant difficulties during their operating. At the same time the development of information technologies allows creating self-learning systems that, by means of neurolinguistic networks, are capable of adjusting to the tasks of an expert-evaluator independently on the basis of peculiarities of market conditions and expertise tasks.

Unfortunately, in modern information systems of expert evaluation of projects we cannot witness the application of technologies of self-developing systems, and, therefore, we reckon that the development of such information system of expert evaluation for each single branch of industry, such as machine building, is prospective and demanding additional theoretical methodological studies [2]. In the framework of this paper we have attempted to study the basic trends of creation of such systems.

Thus, the purpose of the present paper is to develop the basic provisions and theses underlying the creation of a self-learning system of expertise of scientific and industrial projects.

\section{Results and discussion}

Since the scope of the problems and tasks in the field of science and industry is quite wide, in this study we have confined ourselves to a narrower topic. Machine building has been selected as a main application of the expert systems under development. And the basic priority in the creation of the systems was given to orientation to solution of problems of accelerated import substitution and industrial development. According to the authors' view, a modern information system should have neurolinguistic networks as a logic element allowing increasing accuracy and objectivity of the results of their selection in the process of its exploitation by means of adapting the magnitude of weight coefficients of evaluation of scientific projects to the specificity of a realisation field.

Among the main problems that are necessary to solve when creating an effective system of expert selection it is possible to single out:

1. Description of the model of a process of scientific projects expertise oriented to receiving an objective evaluation of their prospectivity and expediency of implementation.

2. Development of valid criteria of evaluation of prospectivity and expediency of projects realisation by means of the analysis of experience of successfully realised projects and projects, being in the process of realisation, at industrial enterprises and in scientific-educational establishments. Justification of criteria of determination of weighty coefficients of significance of evaluation criteria of scientific projects, considering the specific character of heavy engineering.

3. Justification of criteria for determining weight coefficients of significance of criteria of scientific projects evaluation, taking into consideration the specificity of heavy engineering.

4. Development of a programme application for calculation of efficiency of scientific projects.

5. Approbation of the proposed programme at industrial enterprises and at universities.

Creation of expert systems, both in science and in industry, is a theoretical application problem. And such problem is mainly oriented to theoretical methodological description and justification of solution of the problem of enhancement of operationability and efficiency of expertise of scientific projects, realised in the machinebuilding sphere and directed at provision of accelerated import substitution. Formation of expert systems requires specific instruments. Undoubtedly, in order to create a new approach and new breakthrough trends it is necessary to create proper instruments. But it is reasonable to simultaneously use already existing gained experience. The methods and models developed earlier can assist significantly during formation of an expert system. In this case the degree of maturity of already existing methods can vary. Some users apply them as they are, and there are software products already realising these methods. The example of such methods can be the following:

- methodology «Business Process Model and Notation» (BPMN);

- data flow digrams (DFD) for model construction of the expertise process of scientific projects from a position of its transparency and clarity.

BPMN methodology uses conventional signs for depicting business processes in the form of the diagrams of business processes. BPMN is intended for application by both technicians and business users. For this purpose the language uses the core set of intuitive measuring 
elements, which allow defining complex semantic constructions. This methodology is realised in the form of a sufficiently vast set of software products of different producers. All of them are united by a common specification of the use of signs, symbols and so forth.

The main purpose of BPMN is creation of a standard set of conventional signs comprehensible for all business users. BPMN is designed to serve as a link between the phase of business process designing and the phase of its realisation. But such modelling requires a significant amount of time for processing of only one project. In the case of expert evaluation of a considerable quantity of projects, the time expenditures can be extremely great. And for conducting expert evaluations it is efficiently to use only separate elements of this method.

More effective thrashed out methods intended for application in expert systems can be data flow diagrams. Data flow diagrams (DFD) represent a hierarchy of functional processes connected by the data flows. The purpose of such representation is to demonstrate how each process transforms its input data into output ones, as well as to reveal the relations between these processes. In accordance with this method, the system model is determined as a hierarchy of data flow diagrams, describing an asynchronous process of information transformation, beginning from its input into the system up to its delivery to a user. Information sources (external entities) generate information flows (data flows), transferring information to subsystems or processes. They, in their turn, transform information and generate new flows, which transfer information to other processes and subsystems, data storages or external entities - consumers of information. The diagrams of the upper level of the hierarchy (context diagrams) determine basic processes or subsystems equipped with external inputs and outputs. They are detailed by means of the diagram of the lower level [3]. Controlling the degree of decomposition of the general structure it is possible to control both time expenditures on the model construction and the accuracy of model functioning, its correspondence to reality. Reducing the degree of decomposition we not only reduce the time of model construction, but also reduce the degree of its reliability. When using this method it is possible to form some criteria determining the degree of system decomposition during evaluation, which would allow achieving a required degree of reliability and acceptable time expenditures on system construction.

A common drawback of such systems as BPMN and DFD is a considerable proportion of work, performed in order to construct and thrash out the models. When changing the field of application of these models or when changing the priorities, an essential rework of the methods with the help of an operator or developer is required.

However, there are other prospective methods, software and hardware realisation of which for specific tasks can lead to a tangible result. However, their application is associated with the necessity of adaptation to the application conditions. Among such methods, it is possible to specify the following:
- a system approach enabling construction of a structural model of the expertise process of a scientific project, including the description of the main domains of evaluation;

- Delphi technique allowing describing criteria and their weight values;

- artificial neural networks.

In this case, a model of the analysed project is formed with the use of the system approach. Since the system approach is a thrashed out theme possessing a strongly developed methodological apparatus, there is a possibility of its quick adaptation to the conditions of both science and any other industry field [4, 5]. However, in order to compare the projects' models it is necessary to elaborate the evaluation criteria. For this purpose Delphi technique is quite suitable. It allows forming both evaluation criteria and weight values of these criteria with the help of some independent experts (often unconnected and unaware of each other). This method allows avoiding public conflicts between the representatives of the opposite viewpoints as it excludes the immediate contact of experts between each other and, consequently, group influence, arising in the course of teamwork and consisting in adjustment to the majority opinion. In addition, it enables to interview experts extraterritorially without gathering them in one place (for example, by means of electronic mail).

The work performed according to a described method will allow forming an algorithm or programme of project evaluation in a definite field adapted to specific tasks. The drawback of such system will be its static character and orientation to specific conditions. The use of artificial neural networks during development of an evaluation programme will allow "reviving" the system. The opportunity of learning is one of the main advantages of the neural networks over the traditional algorithms. Technically, learning consists in detecting the coefficients of couplings between neurons. In the process of learning, a neural network is capable of revealing complex dependencies between input and output data and of carrying out generalisation.

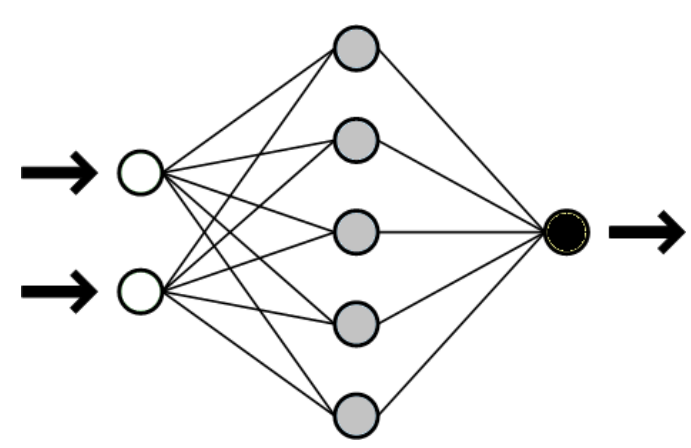

Fig. 1. The scheme of the simplest neural network.

The operation of a neural network is carried out owing to the fact that the neurons are arranged in layers in a regular pattern (Figure 1). The input layer serves just to input the values of input variables. Each of the concealed and output neurons is connected with all elements of the previous layer. We could consider the 
networks where neurons are connected with only some of the neurons of the previous layer; however, for the majority of applications the network having a complete system of connections is preferable, that is, namely this type of networks has been realised in the package "ST Neural Networks"

This implies that in case of successful learning a network can restore a correct result on the basis of data which were absent from the learning sample, as well as on the basis of incomplete or/and "noisy", partially distorted data. In this case, after conducting a number of expert evaluations and formation of the data base of evaluation criteria and weight coefficients according to different conditions of expert evaluation, it will be possible to "teach" the system to generate criteria and weight coefficients in accord with new operating conditions. When reaching a definite level of evaluation data, this will allow obtaining an optimal selection of projects from the viewpoint of set goals with minimal participation of experts.

During development of the common system of expert evaluations, it is expedient to compliment the above-described methods while solving small particular problems. In this case it is possible to use the methods of: economic analysis (development of a system of indexes, comparison method, construction of analytical tables, grouping and detailing methods, calculation of specific rates, analysis of the structure and dynamics of indexes), information collection (the work with information provided in Russian and foreign scientific publications, in analytical materials, in statistical bulletins and editions, in normative-legal documents), and information processing (statistical analysis, expert methods, grouping methods). Combination of these methods will allow developing an effective information system of expertise of scientific projects.

All the work on formation of the system of expert evaluation both in machine building and in any field of application can be conditionally divided by two big stages. The first stage is development of the structure and content of the information system of expert evaluation of scientific projects in the field of machine building industry, including deliberate evaluation criteria, their threshold values-filters, a developed estimation scale and the elements of a self-learning system. The sequence of actions in implementation of this stage can be determined as:

- Application of methodology of the applied system analysis for construction of the model of the content and the structure of the projectable information system. Besides, on the basis of the same methodology it is necessary to develop a greater model - a model of interaction of the information system with all types of users.

- As key methods for formation of interdisciplinary criteria, underlying the operation of the information system, it is necessary to use Delphi technique, morphological analysis, TKJ method and synectics.

- On completion of the procedure of criteria formation, a selection with respect to some project takes place. The selection is carried out on the basis of a supercriterion, constrained optimisation or introduction of an additional criterion.

And the second stage is creation of an integral structure of the self-learning information system of selection of scientific projects of machine building industry, development of a software product, its approbation on the basis of industrial enterprises and in educational establishments. The succession of realisation of this stage will look as follows:

- Constant enhancement of the performance quality of the information system is provided due to neurolinguistic networks.

- Construction of the model of information system functioning is implemented with the use of such notations as IDEF, EPC, Aris and a vector graphics editor "MS Visio".

\section{References}

1. B.C. George, E.N. Teitelbaum, D.A. DaRosa, M. Schuller, J.B.Zwischenberger, Journal of Surgical Education, 70(6), 703-708 (2013)

2. G. Osipov, I. Smirnov, I. Tikhomirov, I. Sochenkov, A. Shelmanov, Studies in Computational Intelligence, 586, 269-285 (2016)

3. A.M. Venderov, CASE-technologies. Modern methods and means of information systems designing (Moscow, Finance and statistics, 2005)

4. Robert K. Merton, Social Theory and Social Structure (Free Press, New York, 1968)

5. K.G. Bardakova, O.M. Goreva, Izvestia visshih uchebnih zavedeniy. Sociology. Economics. Politics, 4, 120-123 (2010) 